\title{
Psychosocial Predictors of Reported Behavior Change in Homosexual Men at Risk for AIDS
}

\author{
Carol-Ann Emmons, PhD \\ Jill G. Joseph, PhD \\ Ronald C. Kessler, PhD \\ Camille B. Wortman, PhD \\ Susanne B. Montgomery, MS \\ David G. Ostrow, MD
}

This article investigates the relationship between psychosocial factors and gay men's at tempts to change their sexual behavior in response to the threat of AIDS. Questionnaire data collected from 909 homosexual men at risk of AIDS were used to quantify potential psychosocial predictors of health behavior. Multiple logistic regression analyses were used to estimate the relationship between these predictor variables and five self-reported behaviors which may reduce transmission of HTLV-III. Knowledge regarding AIDS was consistently and positively related to each of the outcome measures. Perceived risk of AIDS and the perceived efficacy of behavioral changes for reducing one's chances of developing AIDS were also related to multiple outcome measures. Difficulties with sexual impulse control, belief in biomedical technology to prevent/ cure AIDS, and perceived social norms were related only to behaviors which would alter the number or type of one's sexual partners. Gay social network affiliation was not related to any of the outcomes assessed in these analyses. A negative interaction between perceived risk of AIDS and the perceived efficacy of behavioral change was observed for several outcomes. Implications of this work for the development of public policy and educational programs are discussed.

This article investigates the relationship between psychosocial factors and gay men's attempts to change their sexual behavior in response to the threat of AIDS. Over

Carol-Ann Emmons, Jill G. Joseph, Ronald C. Kessler, Camille B. Wortman, Susanne B. Montgomery, and David G. Ostrow are with the University of Michigan, Ann Arbor, Michigan.

This work was supported by research funding from the National Institute of Mental Health (2 R01 MH39346-02A 1) and the University of Michigan.

The authors are grateful to Anita DeLongis for comments made on an earlier draft.

Please refer questions, comments, and requests for reprints to Jill G. Joseph, PhD, Department of Epidemiology, School of Public Health, University of Michigan, 109 Observatory Street, Ann Arbor, MI 48109. 
the past two decades, considerable theoretical and empirical work has examined the relationship between a variety of psychosocial predictors and health-promoting behaviors. $^{1-6}$ This work is based on a recognition that the major sources of morbidity and mortality increasingly have antecedents in patterns of behavior which may be modifiable. ${ }^{7-10}$ This literature may thus help clarify the psychosocial mechanisms influencing behavior in response to the threat of AIDS. Knowledge of these mechanisms is of considerable importance, as public health efforts to limit the spread of AIDS are largely dependent upon behavior changes required to reduce transmission of HTLV-III ${ }^{11,12}$

Although more than a dozen separate models have been proposed to predict health behavior, a recent review demonstrated that they share a common set of six core conceptual elements: knowledge about the disease, perceptions of vulnerability to the disease, beliefs about the efficacy of health care, the accessibility of health care, social network characteristics, and demographic characteristics. ${ }^{13}$ The first of these factors, knowledge, is frequently a prerequisite for behavior change. For example, increased knowledge of cardiovascular risk factors and of appropriate behavioral risk-reduction alternatives has been the goal of both community ${ }^{14}$ and clinic-based intervention programs. ${ }^{15}$ Similarly, knowledge of AIDS and of risk-reduction guidelines would be expected to foster preventive behavior changes in gay men. The gay subculture is being informed of the behavioral risk factors for AIDS through vigorous efforts of their community organizations as well as mass media and informal networks of information sharing. There have been, however, few systematic attempts to evaluate the level of knowledge about AIDS in this population or the effects of such knowledge on behavior. ${ }^{16}$ Thus, little is presently known about the success of educational efforts, or of unmet educational needs that may exist among gay men.

Perceptions of vulnerability to the disease might be expected to play a central role in behavior change in response to AIDS, as considerable evidence is already available linking such perceptions to other health protective behavior. ${ }^{1,17-24}$ Although little is presently known about how such perceptions originate, ${ }^{25}$ recent research suggests that an "optimistic bias" (or underestimation of risk) characterizes beliefs about one's own vulnerability to illness. ${ }^{25-30}$ Evidence that this optimistic bias can be overcome by information comparing personal risk with that of peers, ${ }^{25,31}$ suggests that knowledge of risk factors and objective risk can influence perceptions of risk.

Evaluation of recommended preventive measures also influences the acceptance of a behavioral health recommendation. Evidence from research on community health campaigns indicates that the decision to adopt a preventive health behavior is largely contingent on how effective the behavior is perceived to be in preventing the threatening illness or condition. ${ }^{17,18,24,32-34}$ In the case of AIDS, evidence that many homosexually active men are already HTLV-III seropositive, the long incubation period for AIDS, and the increasing incidence of the syndrome may cause some men to doubt the efficacy of changing their current sexual behavior, especially if they view their past sexual behavior as already placing them at considerable risk.

Access to health or preventive care is known to influence the likelihood that an individual will adopt a desired behavior and is another of the six core conceptual elements identified in the integrated health behavior model. For example, both geographic and financial factors have been demonstrated to effect such outcomes as medical care utilization, ${ }^{5}$ acceptance of screening, ${ }^{35}$ and a general index of health-related behavior. ${ }^{36}$ Several researchers have suggested that in addition to such material barriers to access, a variety of dispositional factors may create behavioral or psychological 
impediments to change. ${ }^{17,37,38}$ Although some authors have reported barriers to health care for gay men reluctant to reveal their homosexuality to physicians, ${ }^{39}$ accessibility to health care is not likely to be important for behavior change to prevent the spread of AIDS as such change does not generally require the assistance of a health care practitioner. However, it would be important to examine dispositional factors which could limit adoption of recommended behavior changes.

Social networks have been defined both in terms of social contact within and between a population subgroup, and in terms of social norms. Past research has demonstrated that individuals with strong social ties are more likely to alter health-threatening behaviors than those with weaker social connections to others. ${ }^{40-43}$ In addition to measures of social contact, social norms are known to influence health behaviors. $^{6,42-44}$ Thus, it is not only the presence of a social network, but behavior and values within the network, which influence health behaviors. Previous research has already demonstrated considerable heterogeneity in social behavior and norms within the homosexual subculture, ${ }^{45,46}$ but the relationship of social networks to preventive health behaviors in this group requires further investigation.

The adoption of preventive health behaviors is also consistently related to certain demographic characteristics. For example, education and income are generally associated with the adoption of preventive health behavior. ${ }^{47}$ Although in large measure unmodifiable, such factors need to be taken into account when attempting to estimate the association between psychosocial factors and behavior changes in response to AIDS. One such factor often explored is marital status; in this cohort participation in a "primary relationship" could be considered as a functional alternative.

While each of the psychosocial factors already described generally predict a variety of health-promoting behaviors, the relative importance of these factors varies depending on the health behavior in question. In planning behavioral interventions to prevent transmission of HTLV-III, it would therefore be useful to compare the various factors in terms of their ability to predict changes in sexual behavior. This article investigates the relationship of these factors to five measures of behavioral responses to the threat of AIDS: (1) any change in behavior; (2) trying to limit the number of one's sexual partners; (3) avoidance of anonymous partners; (4) avoidance of receptive anal intercourse; (5) modification of receptive anal intercourse in ways which may reduce exposure to HTLV-III (asking one's partner to use condoms or withdraw prior to ejaculation). These measures were chosen because they describe a range of behavioral responses from the most general (any change) to the very specific (modifying receptive anal intercourse) and because they include measures which current epidemiologic evidence suggests would most likely reduce exposure to HTLV-III. ${ }^{48}$ Each will be described in greater detail later in this paper.

\section{METHODS}

\section{Subjects}

The sample consisted of 978 homosexual men participating in the Multicenter AIDS Cohort Study (MACS) in Chicago. MACS is a collaborative biomedical study of 
Table 1. Cohort Description: Sociodemographic Characteristics $(N=909)$

\begin{tabular}{lc}
\hline Mean age & 34.6 years \\
Mean education & 16.2 years \\
Mean income "primary relationship" & $\$ 24,794$ \\
Currently in a "primar & $49.5 \%$ \\
\hline
\end{tabular}

the natural history of AIDS funded by the National Institute of Allergic and Infectious Diseases. Respondents were recruited for this study in a number of ways. Approximately $60 \%$ responded to publicity for the study in local gay-oriented periodicals or distributed at a gay clinic: $20 \%$ were recruited from college campuses and gay organizations in the Chicago area; $9 \%$ were formerly enrolled in a Hepatitis B vaccine trial; $5 \%$ were staff, volunteers, and friends of the gay clinic where the study is largely housed; and $6 \%$ came from other sources. such as hearing about the study through word-of-mouth. The sample is predominantly white $(91.1 \%)$, has a mean age of 34.6 years, an average of 16.2 years of education, and 16.5 years of homosexual experience. Although it is not possible to assume that participants are representative of all homosexual men in Chicago, their sociodemographic characteristics suggest that they resemble both homosexual men diagnosed with AIDS in Chicago and cohorts being studied in other metropolitan areas. ${ }^{45}$ The underrepresentation of blacks and His. panics in this and other cohorts should be noted, however. Results described in the present article are confined to those men not diagnosed with AIDS, not being paid for sex, and for whom data were missing on no more than three variables $(n=909)$. Table 1 describes the sociodemographic characteristics of this group. Some analyses focus on restricted subsets of participants. resulting in a reduced number of participants available for study. These subsets are described in greater detail later in this paper.

\section{Procedure}

All participants in the MACS study were invited to enroll in the present psychosocial study. Enrollment took place over a 15-month period (June, 1984-August, 1985). A full 95\% of MACS participants agreed to participate in the psychosocial study and $90 \%$ returned completed questionnaires. Respondents were given a selfadministered questionnaire when they visited the clinic for the MACS study, and asked to complete it two weeks later and mail it back to the clinic. The questionnaire assessed a broad range of psychosocial variables thought to be related to homosexual men's responses to the threat of AIDS. This article focuses on those six core concepts which may be related to men's attempts to change their sexual behavior in response to AIDS. Later in this report we identify and describe predictor and outcome variables used in the analysis.

\section{Psychosocial Assessment}

Table 2 summarizes data regarding each of the variables discussed later. A more detailed description of variable coding procedures will be provided to interested read. 
Table 2. Cohort Description: Psychosocial Characteristics ( $N=909)$

\begin{tabular}{lccc}
\hline Characteristic & $\begin{array}{c}\text { Range } \\
\text { (lowest to highest) }\end{array}$ & Mean & S.D. \\
\hline Knowledge regarding AIDS & $1-7$ & 4.8 & 1.6 \\
$\begin{array}{l}\text { Perceived risk of AIDS } \\
\begin{array}{l}\text { Perceived efficacy of } \\
\quad \text { behavior change }\end{array}\end{array}$ & $1-9$ & 4.0 & 1.7 \\
$\begin{array}{l}\text { Difficulties with sexual } \\
\quad \text { impulse control }\end{array}$ & $1-5$ & 4.1 & 1.2 \\
$\begin{array}{l}\text { Belief in biomedical technology } \\
\quad \text { to prevent/cure AIDS }\end{array}$ & $1-9$ & 3.3 & 1.7 \\
$\begin{array}{l}\text { Perceived social norms } \\
\quad \text { supportive of behavior change }\end{array}$ & $1-3$ & 1.2 & 0.5 \\
Gay social network affiliation & $1-5$ & 3.2 & 1.0 \\
\hline
\end{tabular}

ers upon request. For respondents missing data necessary to code one or two variables, the mean score on those variables was imputed to them. Respondents missing data necessary to code four or more variables were eliminated from the analyses; these constituted less than $1 \%$ of the cohort. For those psychosocial indices were inter-item correlations between score components were, on theoretical grounds, expected to be high, reliability coefficients were calculated. These ranged from 0.52 for a measure of social norms to 0.68 for the perceived risk and knowledge scores. Intercorrelations between the psychosocial indices were also inspected and were found to be modest. ranging from $0.008--0.283$. This finding ensures that multicollinearity is not a serious concern in the analyses reported later in this report.

\section{Knowledge Regarding AIDS}

A knowledge score was computed for each respondent based on seven questions concerning the causal agent for AIDS, modes of transmission for the HTLV-III virus, and public health recommendations regarding behavior changes required to reduce the risk of AIDS. Scores ranged from one to seven, with a mean of 4.8 and a standard deviation of 1.6 .

\section{Perceived Risk}

Two measures of perceived vulnerability to AIDS were obtained from respondents. The first was a measure of absolute risk, based on a question asking men to rate the likelihood of their getting AIDS. The second was a measure of comparative risk, which used a similar question format but asked men to relate how their chances of getting AIDS compared with those of the average gay man. Both questions provided a fivepoint scale. When summed, a general measure of perceived risk for AIDS was obtained with a range from $1-9$, a mean of 4.0 , and a standard deviation of 1.7 . 
Respondents' beliefs about the degree to which behavior change could reduce their chances of getting AIDS was assessed with two questions. All respondents were asked if they did everything they could do to reduce their chances of getting AIDS, how much more this would reduce their risk. Respondents who indicated that they had already changed some aspect of their behavior were also asked how much they thought this had reduced their risk. For the latter respondents, perceived efficacy of behavior change was a composite of their responses to the first and second questions. The first question alone was used as a measure of perceived efficacy for those respondents who said they had not changed their behavior in any way to reduce their risk for AIDS. Scores for perceived efficacy ranged from 1-5 with a mean of 4.1 and a standard deviation of 1.2 .

\section{Barriers to Behavioral Change}

Two concepts were used to assess the presence of potential dispositional barriers to behavior change: (1) perceived difficulty with the control of sexual impulses; (2) belief in biomedical technology to prevent or cure AIDS. The first measure was included because behavioral risk reduction is largely dependent on alteration of sexual practices and perceived difficulties with impulse control might plausibly constitute a barrier to implementing recommended changes. Also, difficulty with sexual impulse control was one of the most frequently identified reasons for continued unsafe behavior in this cohort. ${ }^{45}$ Responses to two questions regarding perceived difficulty in controlling sexual impulses were summed to provide a score on this measure ranging from $1-9$. with a mean of 3.3 , and a standard deviation of 1.7 . The second measure was included because it has been suggested that a belief in biomedical technology to provide a prevention or cure for AIDS may impede the adoption of appropriate behavioral changes in the at-risk gay population. ${ }^{11}$ Two questions specifically assessing respondent beliefs that a vaccine or cure for AIDS would become available in the near future were used to construct this variable. Scores ranged from $1-3$ with a mean of 1.2 and a standard deviation of 0.5 .

\section{Social Network Characteristics}

Two aspects of respondents' social networks were assessed: (1) perceived socia] norms supportive of behavioral change and (2) gay social network affiliation. The questions regarding perceived social norms focused on four specific types of sexual behavior potentially linked to HTLV-III transmission: receptive anal sex, receptive oral-genital sex, insertive oral-anal contact, and use of anonymous partners. Scores for this variable ranged from 1 to 5 , with a mean of 3.2 and a standard deviation of 1.0. Gay social network affiliations were assessed by inquiring about residence in a gay neighborhood, whether at least half the respondent's friends were gay, and whether he was a member in at least one gay organization. Scores ranged from $1-4$, with a mean of 2.6 and a standard deviation of 0.9 . 
Table 3. Self-Reported Behaviors Consistent with Public Health Recommendations Regarding Reduction in HTLV-III Transmission

\begin{tabular}{lccc}
\hline $\begin{array}{c}\text { Time Period } \\
\text { Behavior }\end{array}$ & $\begin{array}{c}\text { Total } \\
N\end{array}$ & $\begin{array}{c}\text { Reporting } \\
\text { Behavior } \\
N(\%)\end{array}$ & $\begin{array}{c}\text { Not } \\
\text { Reporting } \\
\text { Behavior } \\
N(\%)\end{array}$ \\
\hline $\begin{array}{c}\text { Since AIDS epidemic began: } \\
\text { Any behavioral changes } \\
\text { because of AIDS }\end{array}$ & 909 & $732(80.5 \%)$ & $177(19.5 \%)$ \\
$\begin{array}{c}\text { Attempts to reduce number } \\
\text { of sexual partners }\end{array}$ & $867^{\mathrm{a}}$ & $663(76.5 \%)$ & $204(23.5 \%)$ \\
$\begin{array}{c}\text { During the past month: } \\
\begin{array}{c}\text { Avoidance of anonymous } \\
\text { partners because of AIDS }\end{array}\end{array}$ & $591^{\mathrm{b}}$ & $144(24.4 \%)$ & $447(75.6 \%)$ \\
$\begin{array}{c}\text { Avoidance of receptive } \\
\text { anal sex because of AIDS }\end{array}$ & $581^{\mathrm{c}}$ & $75(12.9 \%)$ & $506(87.1 \%)$ \\
$\begin{array}{c}\text { Modification of receptive } \\
\text { anal sex }\end{array}$ & $486^{\mathrm{d}}$ & $98(20.2 \%)$ & $388(79.8 \%)$ \\
\hline
\end{tabular}

aExcludes those who were not homosexually active prior to AIDS epidemic or whose number of partners did not need to be reduced.

bExcludes those who never had anonymous sexual partners or who avoided anonymous partners in the past month for some reason other than AIDS.

cExcludes those who never practiced receptive anal sex or who avoided receptive anal sex in the past month for some reason other than AIDS.

dExcludes those who did not practice receptive anal sex in the past month.

eModification of receptive anal sex is defined by practices which may reduce exposure to HTLV-III: asking partners to use condoms or withdrawing prior to ejaculation.

\section{Behavioral Assessment}

Five dichotomous outcome measures were used in the analyses reported here. Two of these were more global measures describing behavior changes at any time since the beginning of the AIDS epidemic, while the other three described specific sexual behavior during the previous 30 days. The prevalence of these measures is reported in Table 3. It should be noted that only the measure which assessed any behavior change was appropriately calculated in the entire cohort. For each of the other measures, subgroup analyses were performed in order to examine the relevant group of respondents. The number of respondents available for study in these sub-groups ranged from 867 486. Modification of receptive anal sex was defined by two practices which may reduce exposure to HTLV-III: asking sexual partners to use condoms or asking partners to withdraw prior to ejaculation. Respondents who reported engaging in either of these practices were coded as modifying receptive anal sex. The questionnaire also inquired about reasons for avoidance of specific sexual practices among those not engaging in them during the preceding month. Men who reported avoiding either receptive anal sex or anonymous partners for some reason other than concern regarding AIDS were excluded from the analyses reported here. For example, many men reported that sexual activity with anonymous partners was simply an infrequent event which did not happen to occur during the month of reporting. Preliminary analyses 
examined the effects of both including and excluding the group reporting avoidance not explicitly linked to AIDS. As might be expected, greater heterogeneity was introduced by the inclusion of such individuals and the sensitivity of the regression model was decreased. Therefore, analyses reported here focus on specific AIDS-related avoidance of two types of sexual behavior.

\section{Statistical Methods}

Standard multiple logistic regression was used to estimate the relationship between each of the psychosocial variables and the five behavioral outcomes described earlier. The seven psychosocial indices, as well as the sociodemographic variables (age, education, income, and "primary sexual relationship"), were entered simultaneously into the logistic model. Therefore, the coefficients reported later estimate the relationship of each predictor variable on the outcome, while simultaneously taking account of all other varjables.

The possibility that health status might also need to be taken into account in constructing the regression model was investigated in early analyses. Health status in the cohort was measured in a summary score taking account of both lymphadenopathy and a series of specific symptoms. Health was examined both as a predictor variable and as a stratification variable. Its inclusion neither changed the structure of the relationships reported here nor revealed any significant relationship of health itself to outcome. We believe this may reflect the relatively arbitrary criteria used to classify an individual as "unhealthy" in the MACS classification scheme. A specific pattern of lymphadenopathy and two or more symptoms were required to meet case definitions. Therefore, although the unhealthy group is homogeneously ill, by contrast the "healthy" group includes some men who have symptoms. For this reason and because of its lack of importance in predicting the behavioral outcomes, health status is not included as a variable in the analyses reported here.

\section{RESULTS}

Results of the multiple logistic regression analyses are summarized in Table 4 and are discussed in detail later. The relationship of each predictor variable to behavioral outcomes is discussed first, followed by general comments on additional analytic work.

\section{Knowledge Regarding AIDS}

In this cohort of volunteers for a research project focused on AIDS, and with an average 16.2 years of education, knowledge regarding AIDS was generally high. Nonetheless, in terms of both the magnitude and consistency of effects, variation in knowledge regarding AIDS is the factor most strongly associated with the behavioral changes considered here. It is important to note, however, that these data were collected from a single cross-sectional survey. Thus, all that can be reported is an association between knowledge and the desired behavioral outcomes. This point, to be discussed later, 


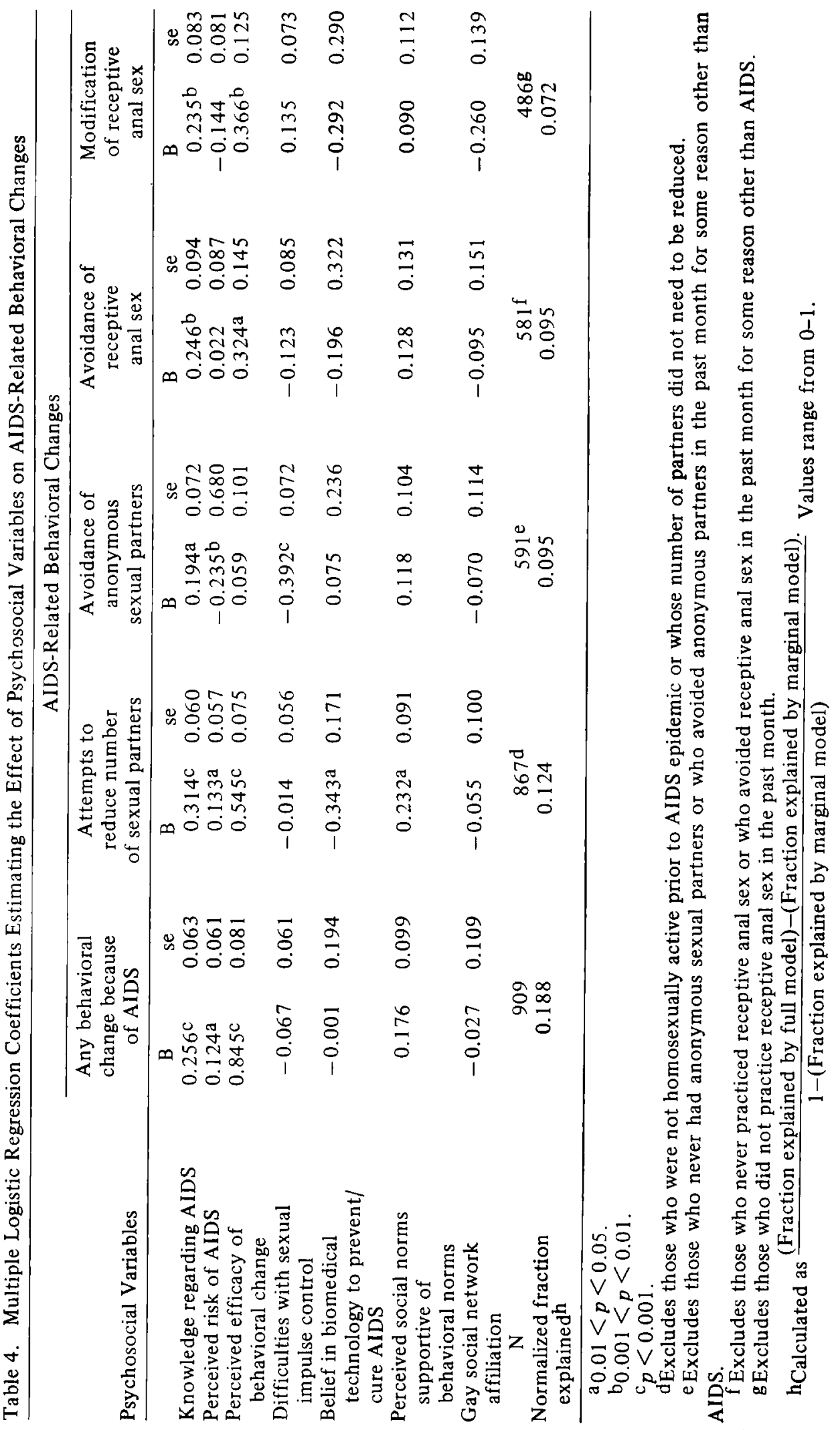


suggests that longitudinal data will be required to clarify the relationships discussed here.

\section{Perceived Risk of AIDS}

Although it is reasonable to expect that perceived risk of AIDS would be associated with the adoption of recommended behavior changes, this was not uniformly true in these analyses. A modest positive relationship $(B=0.124, p<0.05)$ to the global measure of any behavioral change because of AIDS and to attempts to reduce the number of sexual partners $(B=0.133, p<0.05$ ) was observed. On the other hand, a strong negative effect of perceived risk was seen when avoidance of anonymous sexual partners was used as the outcome measure $(B=-0.235, p<0.001)$. Two considerations may explain the absence of a consistent and positive relationship between perceived risk and behavior changes: (1) biased reports of risk and (2) cross-sectional study design.

The "optimistic bias" often observed in the assessment of personal risk was also seen in this cohort. As discussed earlier, although there was considerable variability in the measure of perceived risk, the mean fell below the midpoint of a nine-point scale. More detailed examination of the components of the index further clarify this point. When asked about their absolute risk of developing AIDS, only $10 \%$ of the sample thought they had a large or very large chance of getting AIDS, 50\% thought they had some chance, and the remaining $40 \%$ thought their chances of getting AIDS were small to negligible. Reports of their risk compared to that of other gay men were similarly optimistic and only $15 \%$ rated their chances as higher or much higher. Furthermore, this sense of optimism was also found in those who continued to engage in putatively risky behaviors. This would suggest that the sense of personal risk has a variety of antecedents, that it may be subject to considerable interindividual variability, and that the relationship between perceived risk and behavior is a complex one, probably mediated by several factors unexamined in this analysis.

The cross-sectional nature of the data may also make detection of the true relationship between perceived risk and behavioral measures more difficult. This may explain its negative relationship to avoidance of anonymous sexual partners during the past month. It is likely that participants with anonymous sexual partners realized that this increased their risk of AIDS: thus, the negative coefficient would reflect a rational assessment by those engaging in a risky behavior rather than suggesting that when perceived risk of AIDS is low, men are less likely to have anonymous sexual partners. The bidirectional influence between the two variables would also make it comparatively more difficult to detect the true underlying association between perceived risk and AIDS-related behavior changes.

\section{Perceived Efficacy of Behavioral Change}

Respondents in this cohort were generally very certain that behavior changes can reduce their risk of AIDS; the highest possible score on this measure was 5 and the observed mean was 4.1 . In spite of this generalized certainty, variability in perceived efficacy was related to four of the five AIDS-related behavioral changes described here. 
Interestingly, the magnitude of this relationship, when recalculated as relative risk estimates, was more pronounced for the two global measures of behavioral change occurring any time in the interval since 1981 (any behavioral change $B=0.845, p<$ 0.001 ; attempts to reduce number of sexual partners $B=0.545, p<0.001$ ). In contrast, the relationship of perceived efficacy to the avoidance of receptive anal sex in the past month $(B=0.324, p<0.05)$ and modification of receptive anal sex during the same time period $(B=0.366, p<0.01)$ was more modest. There was no observed relationship between this index and avoidance of anonymous sexual partners.

\section{Barriers to Behavioral Change}

As discussed previously, there were two measures that assessed potential dispositional barriers to behavioral change. The first of these quantified perceived difficulties was sexual impulse control. Generally speaking, few men reported such difficulties and the measure itself was related only to avoiding anonymous sexual partners $(B=$ $-0.392, p<0.001$ ). The negative coefficient suggests that, as expected, those who reported such difficulties were less likely to have avoided anonymous sexual partners during the preceding month.

Another potential barrier to the adoption of safer sexual practices could arise from a belief that biomedical technology will be able to prevent or cure AIDS in the near future. When assessed as already described, such beliefs were negatively associated with attempts to reduce number of sexual partners $(B=-0.343, p<0.05)$. Again, use of cross-sectional data makes it difficult to determine whether this perception plays a part in promoting continued risky sexual practices or represents an after-the-fact rationalization of behavior.

\section{Social Network Characteristics}

There was no significant relationship between the measure of gay social network affiliations and any behavioral outcome. The social network affiliation index took account of residence in a gay neighborhood, gay friendships, and membership in gay organizations. Whether examined separately or in various combinations, none of these measures had any effect on the behavioral measures reported here. These analyses were performed in order to verify that neither the social affiliation index nor any of its component parts had an important effect on behaviors examined here.

Supportive social norms were significantly related to attempts to reduce one's number of sexual partners $(B=0.232, p<0.05)$. This would suggest that insofar as social networks have any effect, it is not mediated by affiliation per se. Rather, it is how one perceives others as behaving or expecting oneself to behave which appears to be important for the behavior changes investigated here.

\section{Interactions}

After inspecting the results reported above, it seemed reasonable to consider the possibility that there might be important interactions between several of the predictor 
variables. The most theoretically plausible of these were interactions between perceived efficacy and risk, and between social norms and social network affiliation. The reasoning in the first case was that perceived efficacy might only be salient among those who believed themselves to be at-risk for AIDS. In the second case, increased consensus regarding behavior change norms was hypothesized to have a differential effect in those who were the most active in gay networks. To examine these possibilities, analyses were re-run including interaction terms between perceived risk of AIDS and the perceived efficacy of behavioral change and between perceived social norms and gay social network affiliation. The latter interaction was consistently insignificant across all five outcomes. Significant effects were observed, though, for the combined effect of perceived risk and perceived efficacy on three measures of outcome: (1) any behavioral change intended to reduce the risk of AIDS $(B=-0.100, p<0.05)$, (2) attempts to reduce the number of one's sexual partners $(B=-0.118, p<0.01)$, and (3) modification of receptive anal $\operatorname{sex}(B=-0.315, p<0.001)$. The negative coefficients can be interpreted to mean that the effect of perceived efficacy on the behavioral outcomes was attenuated at high levels of perceived risk.

This was examined in greater detail in further analyses which stratified perceived risk into a series of categories. This analysis showed that the critical distinction was between those at the highest level of perceived risk and all others (moderate through lowest risk). In the latter group, there was a significant positive effect of perceived risk; the observed coefficients ranged from 0.6 through 0.9 across the three equations and each was significant at the $p<0.01$ level. The comparable coefficients estimating the effect of perceived risk on the outcome measures among those at highest perceived risk were either insignificant or significantly negative. Although it was not possible to explain the significant negative coefficient among those at the highest risk, a general trend was observed here: the relationship between perceived efficacy and behavior change must take perceived risk into account. In all but those who believed themselves to be at highest risk, higher levels of perceived efficacy were positively associated with desirable behavior changes. However, when perceived risk was highest, perceived efficacy no longer operated in such a straightforward manner.

\section{DISCUSSION}

Several important conclusions may be drawn from the work reported here. Knowledge regarding AIDS emerged as a consistently important variable. This is also true when considered in a different way. Logistic coefficients already reported estimate the amount of change in outcome which would be derived from a one unit change in the predictor variable. It is also important to consider how much change is possible in each predictor. For example, the mean score on perceived efficacy in this group is slightly above 4 , while the maximum value is only 5 . This suggests that although perceived efficacy is important in understanding the behavioral measures, relatively little change in this variable is possible. On the other hand, the mean knowledge score is 4.8 , while the maximum value is 7 .

Given this information, it is then possible to quantify the maximum potential behavior change which could be expected in the outcomes if each man's score on that predictor was moved up to the maximum level, holding all other predictors constant. 
Such calculations take into account the relationship between the predictor and outcome variables, the proportion of respondents already reporting the outcome, and the mean level of the predictor variable in the cohort. When viewed in this fashion, education emerges as the most important predictor variable, suggesting that the maximizing education would result in the greatest improvement in the behavioral outcomes considered here. Such calculations, it should be emphasized, assume that the relationships observed here are causal, that it is possible to produce maximum scores on an index, and that such change would be achieved without altering mean scores on all other variables. While reservations could be expressed about each of these assumptions, this exercise nonetheless highlights the importance of looking at the potential impact of a predictor variable in a number of ways.

It should also be noted that the knowledge index was coded to distinguish those men who were certain that a particular factor was related to AIDS from those who, although they were familiar with a particular claim, continued to express uncertainty. Therefore, the present results suggest that as consensus emerges regarding the behavioral risk factors for AIDS, this should be clearly and consistently communicated. Scientific debate, although essential, should not detract from the straightforward communication of factual information. This is a ubiquitous problem in epidemiology; nonetheless, it is important to remind ourselves that debate and the attendant atmosphere of uncertainty it creates may have important consequences for the population at risk.

The need for further scientific information is also highlighted by results describing the relationship between perceived efficacy and behavioral outcomes. Currently, the process leading from infection to the development of AIDS remains obscure, a fact which may diminish men's sense of control over their future health. This difficulty is likely to be particularly acute among those men who find themselves to be already infected with HTLV-III. Information regarding co-factors or intervening processes which might mediate the risk of developing AIDS would be especially important for seropositive individuals in order for them to maintain a belief that behavioral changes can still contribute to their well being. Indeed, as the debate continues about the role of HTLV-III antibody testing in motivating behavior change, it will be important to examine the ways in which this information may or may not contribute positive behavioral outcomes among those at risk. This is especially true as the results reported here suggest that the relationship between perceived efficacy and behavioral outcome is disturbed when perceived risk is exceptionally high. Taken together, these facts suggest that we need to develop educational strategies which inform individuals about risk without depriving them of a sense of both hope and control over their lives.

The relationship between perceived difficulties with sexual impulse control and the avoidance of anonymous sexual partners may underscore the need for a variety of supplemental clinical programs for the at-risk population. It should be emphasized that we did not quantify "objective" problems with sexual impulse control; the index used here instead quantifies perceived difficulty only. It seems likely that such perceived difficulties may arise from a number of distinct sources, including needs for intimacy distorted by homophobic cultural norms, socialization to a particular male role, use of sexual activity to reduce anxiety or depression, and incompletely developed social or transactional skills. Appropriate clinical or supportive services may help develop an improved sense of personal control by addressing such underlying problems. 
Analyses reported here provide some of the first empirical support for a concern originally expressed theoretically regarding belief in biomedical technology as a potential barrier to change. This suggests that, critically important though biomedical research is, the realistic assessment of near-future potentials should be clearly communicated. Inflated promises of vaccine development or premature reports of success for new therapeutic modalities are never appropriate. With regard to AIDS they may provide, however, actual hazards to responsible, behaviorally based prevention efforts. The importance of social norms in these analyses lends support to the view that research on the relationship between social support and health behavior ought to take account not only of social connections between individuals, but of the values communicated within these networks. It also draws attention to the importance of community-based education programs in developing community norms or values supportive of health-affirming sexual behavior. Research reported by this group and others will ultimately be of value in the current crisis insofar as it contributes to the development of such educational efforts.

\section{References}

1. Becker MH, Haefner DP, Kasl SV, et al.: Selected psychosocial models and correlates of individual health-related behaviors. Medical Care 15: 27-46, 1977.

2. Becker MH, Maiman LA: Models of health-related behavior, in Mechanic D (ed): Handbook of Health, Health Care, and the Health Professions. New York. The Free Press, 1983.

3. Rosenstock IM: Historical origins of the health belief model. Health Educ Monogr 2: 328, 1974.

4. Rischbein M, Ajzen I: Belief, Attitude, Intention and Behavior: An Introduction to Theory and Research. Reading, MA, Addison-Wesley, 1975.

5. Andersen R: A behavioral model of families' use of health services. Center for Health Administration Studies. Research Series No. 25. Chicago, University of Chicago, 1968.

6. Fabrega H: Toward a model of illness behavior. Med Care XI(6): 470-484, 1973.

7. Belloc NB: Relationship of health practices and mortality. Prev Med 2:67-81, 1973.

8. Leventhal H, Cleary PD: The smoking problem: A review of the research and theory in behavioral risk modification. Psychol Bull 88(2): 370-405, 1980.

9. Ockene $\mathrm{J}$ : Changes in cigarette smoking behavior in clinical and community trials. Section 7 , Surgeon General's Report, 1984.

10. Kirscht JP: Preventive health behavior: A review of research and issues. Health Psychology 2: 277-302, 1983

11. Osborn JE: Sounding Board. The AIDS Epidemic: Multidisciplinary trouble. New Eng J Med 314: 779-782, 1986.

12. Cummings $\mathrm{KM}$, Becker $\mathrm{MH}$, Maile $\mathrm{MC}$ : Bringing the models together: An empirical approach to combining variables used to explain health actions. $J$ Behav Med 3: 123-145, 1980.

13. Ostrow DG (ed): Biobehavioral Control of AIDS. New York, Irvington Press, 1986.

14. Maccoby N, Farquhar JW, Wood PD, Alexander J: Reducing the risk of cardiovascular disease: Effects of a community-based campaign on knowledge and behavior. $J$ Comm Hlth 3 : 100-114, 1977.

15. Multiple Risk Factor Intervention Trial Research Group: Multiple Risk Factor Intervention Trial. JAMA 248: 1465-1477, 1982.

16. Bye L: A marketing survey of AIDS education in San Francisco, in Ostrow D (ed); Biobehavioral Control of AIDS. New York, Irvington Press, 1986.

17. Cummings KM, Jette AM, Brock BM, et al.: Psychosocial determinants of immunization behavior in a swine influenza campaign. Medical Care 17:639-649, 1979.

18. Kegeles SS: A field experiment attempt to change beliefs and behavior of women in an urban ghet to. J Health Soc Behav 10: 115-124, 1969.

19. Rosenstock IM, Derryberry M, Carringer, BK: Why people fail to seek poliomyelitis vaccination. Pub Health Reports $74:$ 98-105, 1959.

20. Hallal JC: The relationships of health beliefs, health locus of control, and self concept to the practice of breast self examination in adult women. Nurs Res $31: 137-142,1982$. 
21. King JB: The impact of patients' perceptions of high blood pressure on attendance at screening. Soc Sci Med 10: 1079-1091, 1982.

22. Weinberger, M, Greene JY, Marilin JJ, et al.: Health beliefs and smoking behavior. Am J Pub Health 71: 1353-1355, 1981.

23. Tirrell BE, Hart LU: The relationship of health beliefs and knowledge to exercise compliance in patients after coronary bypass. Heart Lung 9: 487-493, 1980.

24. Beck KH: Driving while under the influence of alcohol: Relationship to attitudes and beliefs in a college population. Am J Drug Alcohol Abuse 8: 377-388, 1981.

25. Weinstein ND: Reducing unrealistic optimism about illness susceptibility. Health Psychology 2: 11-20, 1983 .

26. Larwood L: Swine flu: A field study of self-serving biases. J Applied Soc Psychology 8: 283$289,1978$.

27. Robertson LS: Car crashes: Perceived vulnerability and willingness to pay for crash protection. J Comm Health 3: 136-141, 1977.

28. Kirscht JP, Haefner D, Kegeles SS, et al.: A national study of health beliefs. $J$ Health Hum Behav 7: 248-254, 1966.

29. Kirscht JP, Becker MH, Haefner DP, Maiman LA: Effects of threatening communications and mother's health beliefs on weight change in obese children. J Behav Med 1: 147-157, 1978.

30. Becker MH: Patient adherence to prescribed therapies. Med Care 23(5): 539-555, 1985.

31. Haynes RB: Strategies for improving compliance: A methodologic analysis and review, in Sackett DL, Haynes RB (eds): Compliance with Therapeutic Regimens. Baltimore, MD: Johns Hopkins University, 1976.

32. Dabbs JM, Levanthal $\mathrm{H}$ : Effects of varying the recommendations in a fear-arousing communication. J Personality Soc Psychology 4: 525-531, 1966.

33. Berkanovic E, Telesky C, Reeder S: Structural and social psychological factors in the decision to seek medical care for symptoms. Med Care 19:693-709, 1981.

34. Cummings NM, Becker MH, Kirscht JP, et al.: Psychosocial factors affecting adherence to medical regimens in a group of hemodialysis patients. Med Care 20:567-579, 1982.

35. Hochbaum GM: Public Participation in Medical Screening Program: A Sociopsychological Study. Washington, D.C., U.S. Government Printing Office, 1958.

36. Kasl SV, Cobb S: Health behavior, illness behavior, and sick role behavior. II. Sock role behavior. Arch Environ Health 12: 531-541, 1966.

37. Rundall TG, Wheeler JRC: The effect of income on use of preventive care: An evaluation of alternative explanations. J Health Soc Behav 20: 397-406, 1979.

38. Leavitt F: The health belief model and utilization of ambulatory health services. Soc Sci Med 13A: 105-112, 1979 .

39. Ostrow DG: Homosexuality and sexually transmitted diseases, in Holmes KK et al. (eds); Sexually Transmitted Diseases. New York, McGraw-Hill, 1984.

40. Wilhelmson L, Sanne H, Elmfeldt D, et al.: A controlled trial of physical training after myocardial infarction. Prev Med 4: 491-508, 1975.

41. Levanthal H, Cleary PD: The smoking problem: A review of the research and theory in behavioral risk modification. Psychological Bull 88: 370-405, 1980.

42. Moos RH, Mitchell RE: Social network resources and adaptation: a conceptual framework, in Wills T (ed): Basic Process in Helping Relationships. New York, Academic Press, 1982.

43. Norbeck JS: Social support: A model for clinical research and application. Adv Nurs Sci 43-58, 1981.

44. Israel BA: Social networks and health status: Linking theory, research and practice. Patient Counselling Health Edu 4: 65-79, 1982.

45. Ostrow DG, et al.: Sexual behavior change and persistence in homosexual men. Paper presented at the International Conference on Acquired Immunodeficiency Syndrome. Atlanta, Georgia, April, 1985.

46. McKusick L, Horstman W. Coates TJ: AIDS and sexual behavior reported by gay men in San Francisco. Am J Pub Health 75: 493-496, 1985.

47. Hazuda HP, et al.: Ethnic and social class differences relating to prevention of coronary heart disease: The San Antonio Heart Study. Am J Epidemiol 114: 418-426, 1981.

48. DeVita VT, Hellman S, Rosenberg SA: AIDS: Etiology, Diagnosis, Treatment and Prevention. Philadelphia, Lippincott, 1985. 